\title{
Some Fixed Point Theorems in $\varphi-\psi$ weak contraction on Fuzzy Metric Space
}

\author{
R. Krishnakumar ${ }^{1}$, K. Dinesh ${ }^{2 *}$, D. Dhamodharan ${ }^{3}$ \\ ${ }^{1}$ Department of Mathematics,Urumu Dhanalakshmi College, Tiruchirappalli-19, Tamilnadu, India \\ ${ }^{2}$ Department of Mathematics,Urumu Dhanalakshmi College,Tiruchirappalli-19, Tamilnadu, India \\ ${ }^{3}$ Department of Mathematics,Jamal Mohamed College (Autonomous), Tiruchirappalli-20, India \\ *Corresponding Author: dinesh.skksv93@gmail.com, Tel.: +917418865975
}

Available online at: www.isroset.org

Received: 07/May/2018, Revised: 19/May/2018, Accepted: 16/Jun/2018, Online: 30/Jun/2018

Abstract - In this paper, we discuss some results on fixed point theorems in $\varphi-\psi$ weak contraction on fuzzy metric spaces, which are study of generalisation of some existing results are also given in the form of corollary.

Keywords-fuzzy metric space, continuous t-norm, $\varphi-\psi$ weak contraction

\section{INTRODUCTION}

In 1965, Zadeh[17] initiated the concept of fuzzy sets theory. In 1975, Kramosil and Michalek [12] introduced concept of fuzzy metric space. In 1988, Grabiec [6] propounded of the Banach contraction theorem in fuzzy metric spaces. Here after George and Veeramani [5] modified the definition of fuzzy metric spaces from [12]. Many researchers using [5, 6, 13, 14] and developing fuzzy metric spaces see, $[1,9,10]$. In 1984, M.S.Khan, M.Swaleh and S.Sessa[8] discussed Banach fixed point theorem in metric spaces via altering distance function. In 2012, Y.Shen, Dong Qiu and Wei Chen [16] proved fuzzy metric space using altering distance function as follows

$$
\varphi(M(T x, T y, t)) \geq k(t) . \varphi(M(x, y, t)), \forall x, y \in X, t>0,(1.1)
$$

obtained fixed point result for self-mapping of $T$. Recently, many authors using altering distance function and give their contribution in various metric spaces $[3,4]$.

In this paper, we proved some fixed point theorems in $\varphi-\psi$ weak contraction on fuzzy metric spaces, which are our study of generalisation of some existing results.

Definition 1.1 A fuzzy set $\widetilde{A}$ is defined by $\widetilde{A}=\left(x, \mu_{A}(x)\right): x \in A, \mu_{A}(x) \in[0,1]$.In the pair $\left(x, \mu_{A}(x)\right)$, the first element $x$ belongs to the classical set $\mathrm{A}$, the second element $\mu_{\mathrm{A}}(\mathrm{x})$ belongs to the interval $[0,1]$, is called the membership function.

Definition 1.2 A binary operation $*:[0,1] \times[0,1] \rightarrow[0,1]$ is a continuous t-norm if it satisfies the following conditions:

1. * is associative and commutative,

2. $*$ is continuous,

3. $a * 1=a$ for all $a \in[0,1]$,

4. $a * b \leq c * d$ whenever $a \leq c$ and $b \leq d$ for all

$a, b, c, d \in[0,1]$

\section{Example 1.3}

1. Lukasievicz $t$-norm: $a * b=\max (a+b-1,0)$

2. Product $t$-norm: $a * b=a . b$

3. Minimum $t$-norm: $a * b=\min (a, b)$

Definition 1.4 A fuzzy metric space is an ordered triple $(X, M, *)$ such that $X$ is a nonempty set, $*$ is a continuous $t$-norm and $M$ is a fuzzy set on $X \times X \times(0, \infty) \rightarrow[0,1]$ satisfies the following conditions: $\forall x, y, z \in X$ and $s, t>0$

1. $M(x, y, 0)=0, t>0$,

2. $M(x, y, t)=1$ if and only if $x=y, t>0$

3. $M(x, y, t)=M(y, x, t)$ 
4. $M(x, z, t+s) \geq M(x, y, t) * M(y, z, s)$

5. $M(x, y,):.[0, \infty) \rightarrow[0,1]$ is left-continuous.

Then $M$ is called a fuzzy metric on $X$.

Definition 1.5 A fuzzy metric space is an ordered triple such that $\mathrm{X}$ is a non-empty set, $*$ is a continuous $\mathrm{t}$-norm and $\mathrm{M}$ is a conditions: $\forall x, y, z \in X$ and $s, t>0$ fuzzy set on $X \times X \times(0, \infty) \rightarrow[0,1]$ satisfies the following

1. $M(x, y, t)>0, t>0$

2. $M(x, y, t)=1$ if and only if $x=y, t>0$

3. $M(x, y, t)=M(y, x, t)$

4. $M(x, z, t+s) * M(y, z, s) \leq M(x, z, t+s)$

5. $M(x, y,):.(0, \infty) \rightarrow[0,1]$ is continuous.

Then $M$ is called a fuzzy metric on $X$.

Definition 1.6 Let $(X, M, *)$ be a fuzzy metric space, for $t>0$ the open ball $B(x, r, t)$ with a centre $x \in X$ and a radius $0<r<1$ is defined by

$B(x, r, t)=y \in X: M(x, y, t)>1-r$.

A subset $A \subset X$ is called open if for each $x \in A$, there exist $t>0$ and $0<r<1$ such that $B(x, r, t) \subset A$. Let $\tau$ denote the family of all open subsets of $X$. Then $\tau$ is topology on $X$, called the topology induced by the fuzzy metric $M$.

Definition 1.7 Let $(\mathrm{X}, \mathrm{M}, *)$ be a fuzzy metric space

1. A sequence $x_{n}$ in $X$ is said to be convergent to a point $x$ in $(X, M, *)$ if $\lim _{t \rightarrow \infty} M(x, y, t)=1$ for all $t>0$.

2. sequence $x_{n}$ in $X$ is called a Cauchy sequence in $(X, M, *)$, if for each $0<\varepsilon<1$ and $t>0$, there exists $n_{0} \in N$ such that $M\left(x_{n}, x_{m}, t\right)>1-\varepsilon$ for each $n, m \geq n_{0}$

3. A fuzzy metric space in which every Cauchy sequence is convergent is said to be complete.

4. A fuzzy metric space in which every sequence has a convergent subsequence is said to be compact

Lemma 1.8 Let $(X, M, *)$ be a fuzzy metric space. For all $u, v \in X, M(u, v,$.$) is non-decreasing function.$

Proof. If $M(u, v, t)>M(u, v, s)$ for some $0<t<s$.

Then $M(u, v, t) * M(v, v, s-t) \leq M(u, v, s)<M(u, v, t)$,

Thus $M(u, v, t)<M(u, v, t)<M(u, v, t)$,

(since $M(v, v, s-t)=1)$

which is a contradiction

Definition 1.9 A function $\varphi:[0,1] \rightarrow[0,1]$ is called control function or an altering distance function if it satisfies the following properties:

(CF1). $\varphi$ is strictly decreasing and continuous;

(CF2). $\varphi(\lambda) \geq 0, \forall \lambda \neq 1$ if $\varphi(\lambda)=0$ if and only if $\lambda=1$. It is obvious that $\lim _{\lambda \rightarrow 1^{-}} \varphi(\lambda)=\varphi(1)=0$. where $\varphi$ in class of function $\Phi$.

\section{Main Results}

Theorem 2.1 Let $(X, M, *)$ be a complete strong fuzzy metric space with continuous t-norm $*$ and let $T$ is a self-mapping in $X$ such that

$$
\begin{aligned}
\varphi(M(T u, T v, t)) & \leq \varphi(M(u, T u, t))+M(v, T v, t)+M(T u, v, t) \\
& +M(u, T v, t)+M(u, v, t)+\max (M(u, T u, t), M(v, T v, t)) \\
& -\psi(M(u, T u, t)+M(v, T v, t)+M(T u, v, t)+ \\
& M(u, T v, t)+M(u, v, t)+\max (M(u, T u, t), M(v, T v, t)),
\end{aligned}
$$

where $\varphi$ and $\psi$ are altering distance function and ultra altering distance function respectively, $\varphi(t+s) \leq \varphi(t)+\varphi(s)$ for all $t, s \in S$ Then $T$ has a unique fixed point in $X$.

Proof. Let $u$ be any arbitrary point in $X$ and define a sequence $u_{n} \in X$ such that $u_{n+1}=T u_{n}$. Assume that $u_{n+1}=T u_{n}=u_{n}$ for some $n \in \mathbb{N}$, then $u_{n}$ is a fixed point of $T$. 


$$
\begin{aligned}
& \text { Suppose } u_{n+1} \neq u_{n} \text {, put } u=u_{n-1} \text { and } v=u_{n} \text { in equation (2.1) we get } \\
& \varphi\left(M\left(T u_{n-1}, T u_{n}, t\right)\right) \leq \varphi\left(M\left(u_{n-1}, T u_{n-1}, t\right)+M\left(u_{n}, T u_{n}, t\right)+M\left(T u_{n-1}, u_{n}, t\right)\left(M\left(u_{n-1}, T u_{n}, t\right)\right.\right. \\
& +M\left(u_{n-1}, u_{n}, t\right)+\max \left(M\left(u_{n-1}, T u_{n-1}, t\right), M\left(u_{n}, T u_{n}, t\right)\right) \\
& -\psi\left(M\left(u_{n-1}, T u_{n-1}, t\right)+M\left(u_{n}, T u_{n}, t\right)\right. \\
& +M\left(T u_{n-1}, u_{n}, t\right) M\left(u_{n-1}, T u_{n}, t\right)+M\left(u_{n-1}, u_{n}, t\right) \\
& +\max \left(M\left(u_{n-1}, T u_{n-1}, t\right), M\left(u_{n}, T u_{n}, t\right)\right) \\
& \leq \varphi\left(M\left(u_{n-1}, u_{n}, t\right)\right)+\left(M\left(u_{n}, u_{n+1}, t\right)\right)+\left(M\left(u_{n}, u_{n}, t\right)\right)+\left(M\left(u_{n-1}, u_{n+1}, t\right)\right)+\left(M\left(u_{n-1}, u_{n}, t\right)\right) \\
& +\max \left(M\left(u_{n-1}, u_{n}, t\right), M\left(u_{n}, u_{n+1}, t\right)\right)-\psi\left(M\left(u_{n-1}, u_{n}, t\right)\right)+\left(M\left(u_{n}, u_{n+1}, t\right)\right)+\left(M\left(u_{n}, u_{n}, t\right)\right) \\
& +\left(M\left(u_{n-1}, u_{n+1}, t\right)\right)+\left(M\left(u_{n-1}, u_{n}, t\right)\right)+\max \left(M\left(u_{n-1}, u_{n}, t\right), M\left(u_{n}, u_{n+1}, t\right)\right) \\
& \leq \varphi\left(M\left(u_{n-1}, u_{n}, t\right)\right)+\left(M\left(u_{n}, u_{n+1}, t\right)\right)+\left(M\left(u_{n}, u_{n}, t\right)\right)+\left(M\left(u_{n-1}, u_{n+1}, t\right)\right)+\left(M\left(u_{n-1}, u_{n}, t\right)\right) \\
& +\max \left(M\left(u_{n-1}, u_{n}, t\right), M\left(u_{n}, u_{n+1}, t\right)\right)-\psi\left(M\left(u_{n-1}, u_{n}, t\right)\right)+\left(M\left(u_{n}, u_{n+1}, t\right)\right)+\left(M\left(u_{n}, u_{n}, t\right)\right) \\
& +\left(M\left(u_{n-1}, u_{n+1}, t\right)\right)+\left(M\left(u_{n-1}, u_{n}, t\right)\right)+\max \left(M\left(u_{n-1}, u_{n}, t\right), M\left(u_{n}, u_{n+1}, t\right)\right) \\
& \leq \varphi\left(M\left(u_{n-1}, u_{n}, t\right)\right)+\left(M\left(u_{n}, u_{n+1}, t\right)\right)+\left(M\left(u_{n-1}, u_{n+1}, t\right)\right)+\left(M\left(u_{n-1}, u_{n}, t\right)\right) \\
& +\max \left(M\left(u_{n-1}, u_{n}, t\right), M\left(u_{n}, u_{n+1}, t\right)\right)-\psi M\left(u_{n-1}, u_{n}, t\right)+\left(M\left(u_{n}, u_{n+1}, t\right)\right) \\
& +\left(M\left(u_{n-1}, u_{n+1}, t\right)\right)+\left(M\left(u_{n-1}, u_{n}, t\right)\right)+\max \left(M\left(u_{n-1}, u_{n}, t\right), M\left(u_{n}, u_{n+1}, t\right)\right) \\
& \varphi\left(M\left(u_{n}, u_{n+1}, t\right)\right) \leq \varphi\left(M\left(u_{n-1}, u_{n}, t\right)\right)+\varphi\left(M\left(u_{n}, u_{n+1}, t\right)\right)+\varphi\left(M\left(u_{n-1}, u_{n+1}, t\right)\right)+\varphi\left(M\left(u_{n-1}, u_{n}, t\right)\right) \\
& +\varphi \max \left(M\left(u_{n-1}, u_{n}, t\right), M\left(u_{n}, u_{n+1}, t\right)\right)
\end{aligned}
$$

Here $(X, M, *)$ is a strong fuzzy metric space then we have $M\left(u_{n-1}, u_{n+1}, t\right) \geq M\left(u_{n-1}, u_{n}, t\right) * M\left(u_{n}, u_{n+1}, t\right)$ (by using (GV4'))

$$
\begin{array}{r}
\varphi\left(M\left(u_{n-1}, u_{n+1}, t\right)\right) \geq \varphi\left(\left(M\left(u_{n-1}, u_{n}, t\right)\right) *\left(M\left(u_{n}, u_{n+1}, t\right)\right)\right) \\
\varphi\left(M\left(u_{n-1}, u_{n+1}, t\right)\right) \geq \varphi\left(\left(M\left(u_{n-1}, u_{n}, t\right)\right)+\left(M\left(u_{n}, u_{n+1}, t\right)\right)\right)
\end{array}
$$

Using above inequalities in (2.3) we get

$$
\begin{aligned}
\varphi\left(M\left(u_{n}, u_{n+1}, t\right)\right) & \leq \varphi\left(\left(M\left(u_{n-1}, u_{n}, t\right)\right)+\left(M\left(u_{n}, u_{n+1}, t\right)\right)\right. \\
& +\left[\left(M\left(u_{n-1}, u_{n}, t\right)\right)+\left(M\left(u_{n}, u_{n+1}, t\right)\right)\right] \\
& \left.+\left(M\left(u_{n-1}, u_{n}, t\right)\right)+\max \left(M\left(u_{n-1}, u_{n}, t\right), M\left(u_{n}, u_{n+1}, t\right)\right)\right) \\
& -\psi\left(\left(M\left(u_{n-1}, u_{n}, t\right)\right)+\left(M\left(u_{n}, u_{n+1}, t\right)\right)\right. \\
& +\left[\left(M\left(u_{n-1}, u_{n}, t\right)\right)+\left(M\left(u_{n}, u_{n+1}, t\right)\right)\right] \\
& \left.+\left(M\left(u_{n-1}, u_{n}, t\right)\right)+\max \left(M\left(u_{n-1}, u_{n}, t\right), M\left(u_{n}, u_{n+1}, t\right)\right)\right) \\
\varphi\left(M\left(u_{n}, u_{n+1}, t\right)\right) \leq & \varphi\left(M\left(u_{n-1}, u_{n}, t\right)\right)+\varphi\left(M\left(u_{n}, u_{n+1}, t\right)\right) \\
& +\varphi\left(M\left(u_{n-1}, u_{n}, t\right)\right)+\varphi\left(M\left(u_{n}, u_{n+1}, t\right)\right) \\
& +\varphi\left(M\left(u_{n-1}, u_{n}, t\right)\right)+\varphi \max \left(M\left(u_{n-1}, u_{n}, t\right), M\left(u_{n}, u_{n+1}, t\right)\right)
\end{aligned}
$$

If $\max \left(M\left(u_{n-1}, u_{n}, t\right), M\left(u_{n}, u_{n+1}, t\right)\right)=M\left(u_{n-1}, u_{n}, t\right)(2.6)$

Then the above inequality (2.4) becomes

$$
\begin{aligned}
\varphi\left(M\left(u_{n}, u_{n+1}, t\right)\right) & \leq \varphi\left(M\left(u_{n-1}, u_{n}, t\right)\right)+\varphi\left(M\left(u_{n}, u_{n+1}, t\right)\right) \\
& +\varphi\left(M\left(u_{n-1}, u_{n}, t\right)\right)+\varphi\left(M\left(u_{n}, u_{n+1}, t\right)\right) \\
& +\varphi\left(M\left(u_{n-1}, u_{n}, t\right)\right)+\varphi\left(M\left(u_{n-1}, u_{n}, t\right)\right)
\end{aligned}
$$

Continuing this process, we get,

Similarly,

$$
\varphi\left(M\left(u_{n}, u_{n+1}, t\right)\right) \leq \varphi\left(M\left(u_{n-1}, u_{n}, t\right)\right)<\varphi\left(M\left(u_{n-1}, u_{n}, t\right)\right)
$$

Then the inequality (2.5) becomes

$$
\text { If } \max \left(M\left(u_{n-1}, u_{n}, t\right), M\left(u_{n}, u_{n+1}, t\right)\right)=M\left(u_{n}, u_{n+1}, t\right)
$$

Hence $\varphi\left(M\left(u_{n}, u_{n+1}, t\right)\right)<\varphi\left(M\left(u_{n-1}, u_{n}, t\right)\right)$

$$
\varphi\left(M\left(u_{n}, u_{n+1}, t\right)\right) \leq \varphi\left(M\left(u_{n-1}, u_{n}, t\right)\right)<\varphi\left(M\left(u_{n-1}, u_{n}, t\right)\right)
$$

This gives $\left(M\left(u_{n}, u_{n+1}, t\right)\right)>\left(M\left(u_{n-1}, u_{n}, t\right)\right)$ 
Since the sequence $M\left(u_{n}, u_{n+1}, t\right)$ is non decreasing

Taking limit $n \rightarrow \infty$, we get

$$
\lim _{n \rightarrow \infty} M\left(u_{n}, u_{n+1}, t\right)=q(r), \text { for } q:(0, \infty) \rightarrow[0,1]
$$

Suppose that $q(r) \neq 1$ for some $r>0$ as $n \rightarrow \infty$,

Now (2.7) becomes,

which is a contradiction.

$$
\varphi(q(r)) \leq \varphi(q(r))<\varphi(q(r))
$$

Hence $\lim _{n \rightarrow \infty} M\left(u_{n}, u_{n+1}, t\right)=1, t>0$

Next we prove that the sequence $u_{n}$ is a Cauchy's sequence.

Assume that $u_{n}$ is not a Cauchy's sequence then for any $0<\varepsilon<1, t>0$ then there exists sequence $u_{n_{k}}$ and $u_{m_{k}}$ where $n_{k}, m_{k} \geq n$ and $n_{k}, m_{k} \in \mathbb{N}\left(n_{k}>m_{k}\right)$

$$
\text { suchthat } M\left(u_{n_{k}}, u_{m_{k}}, t\right) \leq 1-\varepsilon
$$

Let $n_{k}$ be least integer exceeding $m_{k}$ satisfying the above property

$$
\therefore \quad M\left(u_{n_{k}-1}, u_{m_{k}}, t\right)>1-\varepsilon, n_{k}, m_{k} \in \mathbb{N} \text { and } t>0
$$

$$
\begin{aligned}
& \text { Put } u=u_{n_{k}-1} \text { and } v=u_{m_{k}-1} \\
& \varphi\left(M\left(T u_{n_{k}-1}, T u_{m_{k}-1}, t\right)\right) \leq \varphi\left(\left(M\left(u_{n_{k}-1}, T u_{n_{k}-1}, t\right)\right)+\left(M\left(u_{m_{k}-1}, T u_{m_{k}-1}, t\right)\right)\right. \\
& +\left(M\left(T u_{n_{k}-1}, u_{m_{k}-1}, t\right)\right)\left(M\left(u_{n_{k}-1}, T u_{m_{k}-1}, t\right)\right) \\
& +\left(M\left(u_{n_{k}-1}, u_{m_{k}-1}, t\right)\right)+ \\
& \left.\max \left(M\left(u_{n_{k}-1}, T u_{n_{k}-1}, t\right), M\left(u_{m_{k}-1}, T u_{m_{k}-1}, t\right)\right)\right) \\
& -\psi\left(\left(M\left(u_{n_{k}-1}, T u_{n_{k}-1}, t\right)\right)+\left(M\left(u_{m_{k}-1}, T u_{m_{k}-1}, t\right)\right)\right. \\
& +\left(M\left(T u_{n_{k}-1}, u_{m_{k}-1}, t\right)\right)\left(M\left(u_{n_{k}-1}, T u_{m_{k}-1}, t\right)\right) \\
& +\left(M\left(u_{n_{k}-1}, u_{m_{k}-1}, t\right)\right)+ \\
& \left.\max \left(M\left(u_{n_{k}-1}, T u_{n_{k}-1}, t\right), M\left(u_{m_{k}-1}, T u_{m_{k}-1}, t\right)\right)\right) \\
& \varphi\left(M\left(u_{n_{k}}, u_{m_{k}}, t\right)\right) \leq \varphi\left(\left(M\left(u_{n_{k}-1}, u_{n_{k}}, t\right)\right)+\left(M\left(u_{m_{k}-1}, u_{m_{k}}, t\right)\right)\right. \\
& +\left(M\left(u_{n_{k}}, u_{m_{k}-1}, t\right)\right)\left(M\left(u_{n_{k}-1}, u_{m_{k}}, t\right)\right) \\
& +\left(M\left(u_{n_{k}-1}, u_{m_{k}-1}, t\right)\right) \\
& \left.+\max \left(M\left(u_{n_{k}-1}, u_{n_{k}}, t\right), M\left(u_{m_{k}-1}, u_{m_{k}}, t\right)\right)\right) \\
& -\psi\left(\left(M\left(u_{n_{k}-1}, u_{n_{k}}, t\right)\right)+\left(M\left(u_{m_{k}-1}, u_{m_{k}}, t\right)\right)\right. \\
& +\left(M\left(u_{n_{k}}, u_{m_{k}-1}, t\right)\right)\left(M\left(u_{n_{k}-1}, u_{m_{k}}, t\right)\right) \\
& +\left(M\left(u_{n_{k}-1}, u_{m_{k}-1}, t\right)\right) \\
& \left.+\max \left(M\left(u_{n_{k}-1}, u_{n_{k}}, t\right), M\left(u_{m_{k}-1}, u_{m_{k}}, t\right)\right)\right)
\end{aligned}
$$


then we get,

$$
\begin{aligned}
\varphi\left(M\left(u_{n_{k}}, u_{m_{k}}, t\right)\right) & \leq \varphi\left(\left(M\left(u_{n_{k}-1}, u_{n_{k}}, t\right)\right)+\left(M\left(u_{m_{k}-1}, u_{m_{k}}, t\right)\right)\right. \\
& +\left(M\left(u_{n_{k}}, u_{m_{k}-1}, t\right)\right)\left(M\left(u_{n_{k}-1}, u_{m_{k}}, t\right)\right)+ \\
& \left.+\left(M\left(u_{n_{k}-1}, u_{m_{k}-1}, t\right)\right)+\left(M\left(u_{n_{k}-1}, u_{n_{k}}, t\right)\right)\right) \\
& -\psi\left(\left(M\left(u_{n_{k}-1}, u_{n_{k}}, t\right)\right)+\left(M\left(u_{m_{k}-1}, u_{m_{k}}, t\right)\right)\right. \\
& \left(M\left(u_{n_{k}}, u_{m_{k}-1}, t\right)\right)\left(M\left(u_{n_{k}-1}, u_{m_{k}}, t\right)\right) \\
& \left.+\left(M\left(u_{n_{k}-1}, u_{m_{k}-1}, t\right)\right)+\left(M\left(u_{n_{k}-1}, u_{n_{k}}, t\right)\right)\right) \\
& \leq \varphi\left(\left(M\left(u_{n_{k}-1}, u_{n_{k}}, t\right)\right)+\left(M\left(u_{m_{k}-1}, u_{m_{k}}, t\right)\right)\right. \\
& +\left(M\left(u_{n_{k}}, u_{m_{k}-1}, t\right)\right)\left(M\left(u_{n_{k}-1}, u_{m_{k}}, t\right)\right) \\
& \left.+\left(M\left(u_{n_{k}-1}, u_{m_{k}-1}, t\right)\right)+\left(M\left(u_{n_{k}-1}, u_{n_{k}}, t\right)\right)\right) \\
& -\psi\left(\left(M\left(u_{n_{k}-1}, u_{n_{k}}, t\right)\right)+\left(M\left(u_{m_{k}-1}, u_{m_{k}}, t\right)\right)\right. \\
& +\left(M\left(u_{n_{k}}, u_{m_{k}-1}, t\right)\right)\left(M\left(u_{n_{k}-1}, u_{m_{k}}, t\right)\right) \\
& \left.+\left(M\left(u_{n_{k}-1}, u_{m_{k}-1}, t\right)\right)+\left(M\left(u_{n_{k}-1}, u_{n_{k}}, t\right)\right)\right) \\
& \leq \varphi\left(M\left(u_{n_{k}-1}, u_{n_{k}}, t\right)\right)+\varphi\left(M\left(u_{m_{k}-1}, u_{m_{k}}, t\right)\right) \\
& +\varphi\left(M\left(u_{n_{k}}, u_{m_{k}-1}, t\right)\right) \varphi\left(M\left(u_{n_{k}-1}, u_{m_{k}}, t\right)\right) \\
& +\varphi\left(M\left(u_{n_{k}-1}, u_{m_{k}-1}, t\right)\right)+\varphi\left(M\left(u_{n_{k}-1}, u_{n_{k}}, t\right)\right)
\end{aligned}
$$

Applying the previous inequalities we get

$$
\varphi\left(M\left(u_{n_{k}}, u_{m_{k}-1}, t\right)\right) \leq \varphi\left(M\left(u_{n_{k}}, u_{m_{k}}, t\right)\right)+\varphi\left(M\left(u_{m_{k}}, u_{m_{k}-1}, t\right)\right)
$$

$$
\operatorname{and} \varphi\left(M\left(u_{n_{k}-1}, u_{m_{k}-1}, t\right)\right) \leq \varphi\left(M\left(u_{n_{k}-1}, u_{n_{k}}, t\right)\right)+\varphi\left(M\left(u_{n_{k}}, u_{m_{k}-1}, t\right)\right)
$$

$$
\begin{aligned}
\varphi\left(M\left(u_{n_{k}-1}, u_{m_{k}-1}, t\right)\right) & \leq \varphi\left(M\left(u_{n_{k}-1}, u_{n_{k}}, t\right)\right)+\varphi\left(M\left(u_{n_{k}}, u_{m_{k}}, t\right)\right)+ \\
& \varphi\left(M\left(u_{m_{k}}, u_{m_{k}-1}, t\right)\right)
\end{aligned}
$$

Also (2.13) and (CF1) we get

$$
\varphi\left(M\left(u_{n_{k}-1}, u_{m_{k}}, t\right)\right) \leq \varphi(1-\varepsilon) .
$$

Substituting (2.16), (2.17), and (2.18) in (2.15) we have

$$
\begin{aligned}
\varphi\left(M\left(u_{n_{k}}, u_{m_{k}}, t\right)\right) \leq & \leq\left(M\left(u_{n_{k}-1}, u_{n_{k}}, t\right)+\left(M\left(u_{m_{k}-1}, u_{m_{k}}, t\right)\right)\right. \\
& +\left(M\left(u_{n_{k}}, u_{m_{k}}, t\right)\right)+\left(M\left(u_{m_{k}}, u_{m_{k}-1}, t\right)\right)(1-\varepsilon) \\
& +\left(M\left(u_{n_{k}-1}, u_{n_{k}}, t\right)\right)+\left(M\left(u_{n_{k}}, u_{m_{k}}, t\right)\right) \\
& \left.+\left(M\left(u_{m_{k}}, u_{m_{k}-1}, t\right)\right)+\left(M\left(u_{n_{k}-1}, u_{n_{k}}, t\right)\right)\right) \\
& -\psi\left(M\left(u_{n_{k}-1}, u_{n_{k}}, t\right)+\left(M\left(u_{m_{k}-1}, u_{m_{k}}, t\right)\right)\right. \\
& +\left(M\left(u_{n_{k}}, u_{m_{k}}, t\right)\right)+\left(M\left(u_{m_{k}}, u_{m_{k}-1}, t\right)\right)(1-\varepsilon) \\
& +\left(M\left(u_{n_{k}-1}, u_{n_{k}}, t\right)\right)+\left(M\left(u_{n_{k}}, u_{m_{k}}, t\right)\right) \\
& \left.+\left(M\left(u_{m_{k}}, u_{m_{k}-1}, t\right)\right)+\left(M\left(u_{n_{k}-1}, u_{n_{k}}, t\right)\right)\right) \\
\varphi\left(M\left(u_{n_{k}}, u_{m_{k}}, t\right)\right) \leq & \varphi\left(M\left(u_{n_{k}-1}, u_{n_{k}}, t\right)\right. \\
+ & \left.\left(M\left(u_{m_{k}}, u_{m_{k}-1}, t\right)\right) \varphi(1-\varepsilon)\right)
\end{aligned}
$$

Using (2.12) we obtain,

$$
\begin{gathered}
\varphi\left(M\left(u_{n_{k}}, u_{m_{k}}, t\right)\right)>\varphi(1-\varepsilon) \\
\varphi(1-\varepsilon) \quad \leq \varphi\left(M\left(u_{n_{k}-1}, u_{n_{k}}, t\right)\right. \\
\left.\quad+\left(M\left(u_{m_{k}}, u_{m_{k}-1}, t\right)\right) \varphi(1-\varepsilon)\right)
\end{gathered}
$$

Taking $k \rightarrow \infty$ in above inequality we obtain

$$
\varphi(1-\varepsilon) \leq \varphi(1-\varepsilon)
$$


That is $\varphi(1-\varepsilon) \leq 0$,

Which is a contradiction, $\varepsilon>0$

Hence $u_{n}$ is a Cauchy's sequence.

Since $X$ is complete and there exist $z \in X$ such that $\lim _{n \rightarrow \infty} u_{n}=z$

That is $M\left(u_{n}, z, t\right)=1$ as $n \rightarrow \infty$

Put $u=u_{n-1}$ and $v=z$ in equation (2.1) we get

$$
\begin{aligned}
\varphi\left(M\left(u_{n}, T z, t\right)\right) & \leq \varphi\left(M\left(u_{n-1}, u_{n}, t\right)+(M(z, T z, t))\right. \\
& +\left(M\left(u_{n}, z, t\right)\right)+\left(M\left(u_{n-1}, T z, t\right)\right) \\
& \left.+\left(M\left(u_{n-1}, z, t\right)\right)+\max \left(M\left(u_{n-1}, u_{n}, t\right), M(z, T z, t)\right)\right)
\end{aligned}
$$

Taking $n \rightarrow \infty$ in (2.23)

Therefore, $M(z, T z, t)=1$, and $z=T z$.

$$
\varphi(M(z, T z, t)) \leq 0, t>0
$$

To prove Uniqueness,

Suppose that $w$ is another fixed point of $T$, that is $T w=w$ where $q \neq z$

Hence $z=w$ is the unique fixed point of $T$.

$$
\varphi(M(z, w, t)) \leq 0, t>0
$$

Corollary 2.2 Let $(X, M, *)$ be a complete strong fuzzy metric space with continuous $t$-norm $*$ and let $T$ is a self-mapping in $X$. If there exists a control function $\varphi$ and $=(t)$, such that

$$
\begin{aligned}
\varphi(M(T u, T v, t)) & \leq \varphi(M(u, T u, t))+(M(v, T v, t))+(M(T u, v, t))+(M(u, T v, t))+(M(u, v, t)) \\
& -\psi(M(u, T u, t))+(M(v, T v, t))+(M(T u, v, t))+(M(u, T v, t))+(M(u, v, t))
\end{aligned}
$$

Then $T$ has a unique fixed point in $X$.

Proof. The proof of the above theorem (2.1) considering the fuzzy contraction on the fuzzy metric space $(X, M, *)$,

$$
\begin{aligned}
\varphi(M(T u, T v, t)) & \leq \varphi(M(u, T u, t)+(M(v, T v, t)) \\
& +(M(T u, v, t))+(M(u, T v, t))+(M(u, v, t))) \\
& -\psi(M(u, T u, t)+(M(v, T v, t)) \\
& +(M(T u, v, t))+(M(u, T v, t))+(M(u, v, t))) .
\end{aligned}
$$

\section{REFERENCE}

[1] C. T. Aage, Binayak S. Choudhury and Krishnapada Das, Some fixed point results in fuzzy metric spaces using control function, Surveys in Mathematics and its Applications, 12 (2017), 23 -34.

[2] K.Amit, and K.V Ramesh, Common fixed point theorem in fuzzy metric space using control function, Commun. Korean Math. Soc. 28 (2013), 517-526.

[3] Arslan Hojat Ansari, R. Krishnakumar and D.Dhamodharan, Common Fixed Point of Four Mapping with Contractive Modulus on Cone Banach Space via cone C-class function, Global Journal of Pure and Applied Mathematics, 13(9) (2017), 5593-5610.

[4] D.Dhamodharan and R. Krishnakumar, Cone S-Metric Space and Fixed Point Theorems of Contractive mappings, Annals of Pure and Applied Mathematics, 14(2)(2017), 237-243.

[5] A.George, and P.Veeramani, On some results in fuzzy metric spaces, Fuzzy Sets and Systems, 64 (1994), 395-399.

[6] A.George, and P.Veeramani, On some results of analysis for fuzzy metric spaces, Fuzzy Sets and Systems, 90 (1997), 365-399.

[7] M.Grabiec, Fixed points in fuzzy metric spaces, Fuzzy Sets and Systems, 27 (1988), 385-389.

[8] V.Gregori, Samuel Morillas, and Almanzor Sapena, Examples of fuzzy metrics and applications, Fuzzy Sets and Systems, 170 (2011), 95-111.

[9] Hsien-Chung Wu, Common Coincidence Points and Common Fixed Points in Fuzzy Semi-Metric Spaces, Mathematics, 6 (29), (2018), 1-21

[10] Humaira, Muhammad Sarwar, and G. N. V. Kishore, Fuzzy Fixed Point Results For $\phi$ Contractive Mapping with Applications, Hindawi Complexity,2018, (2018) 12 pages, doi.org/10.1155/2018/5303815

[11] M.S.Khan, M.Swaleh and S.Sessa, Fixed point theorems by altering distance between the points, Bull. Aust. Math. Soc., 30(1984), 1-9.

[12] J.Kramosil, and J.Michalek, Fuzzy metric and Statistical metric spaces, Kybernetica, 11 (1975), 326-334.

[13] R.Krishnakumar, K. Dinesh, Fixed Point Theorems of Fuzzy Function in Complete Metric Spaces, International Journal Of Innovative Research In Science, Engineering And Technology, 6(7), (2017), 13557-13562

[14] R. Krishnakumar, K. Dinesh, Fuzzy Mapping and their Fixed Point Theorems in Complete Metric Spaces, International Journal of Scientific Research in Science, Engineering and Technology, 3(8) (2017), 241-249

[15] B.Schweizer, and A.Sklar, Statistical metric spaces, Pacific Journal of Mathematics, 10 (1960), 313-334. 
[16] Y.Shen, Dong Qiu and Wei Chen, Fixed point theorems in fuzzy metric spaces, Applied mathematics Letters, 25 (2012), 138-141

[17] Krishnadhan Sarkar, Kalishankar Tiwary, "Common Fixed Point Theorems for Wealkly Compatible mapping on Cone Banach Space" IJSRMSS, Volume 5, issu-2,pp-75-79 (April 2018)

[18] L.A.Zadeh, Fuzzy sets, Inform. Control, 8 (1965), 338-353.

\section{AUTHORS PROFILE}

Dr. R. Krishnakumar is the Associate Professor and Head of the PG \& Research Department of Mathematics, Urumu Dhanalakshmi College. He has above 22 years experience in teaching. His field of research is Fixed point theory.

K. Dinesh is pursuing his Ph.D in PG \& Research Department of Mathematics, Urumu Dhanalakshmi College, Trichy. His field of research is Fixed point theory.

D.Dhamodharan is the assistant professor, Department of mathematics, Jamal Mohamad college, Trichy 